\title{
Novel Control Technique for Control of Circulating Current in Modular Multilevel Converters
}

\author{
Farhan $\mathrm{Ali}^{1}$, Dr Tanvir Ahmad ${ }^{2}$, Asif Khan ${ }^{3}$, Saad Rashid ${ }^{4}$ \\ ${ }^{1,2,3,4}$ US Pakistan Centre for Advance Studies in Energy, University of Engineering and Technology Peshawar, \\ Pakistan. \\ farhann_ali42@yahoo.com ${ }^{1}$, tanvir.ahmad@uetpeshawar.edu.pk ${ }^{2}$, asifengruet@outlook.com, ${ }^{3}$ \\ saad.789@outlook.com ${ }^{4}$ \\ Received: 11 May, Revised: 20 May, Accepted: 25 May
}

\begin{abstract}
Modular multilevel converters are the prominent candidates for high voltage direct current transmission systems. They offer high flexibility, modularity and flexibility in their operation. The main problem of multilevel converters are the circulating current in the converter and arm's voltage balancing in steady state and dynamic state. An easy and flexible control scheme is introduced in this study which eliminates the even order harmonics in the circulating current and reduces the circulating current. The model is implemented in Simulink/Matlab and the results confirm the efficiency of the proposed model., the response of the controller is also presented in result section, which manifest its ability to control the current and eliminate the even order harmonics.
\end{abstract}

Keywords - Modular Multilevel converters, circulating current, Even order harmonics, repetitive controller, arm voltages.

\section{INTRODUCTION}

Energy is the first and foremost need of mankind. Man has needed energy since the beginning in one form or another. The need for energy increased with the advent of industrialization with more energy being needed to cope with the increased demand. Energy is often generated at remote places and then connected to residential and domestic load centers. With the increased demand, efforts started to minimize the losses in energy transmission. The shift from alternating current transmission to direct current transmission system was the culmination of these efforts. At load centers, a modular multilevel converter, converts power from ac to dc which is then transmitted over a long distance. At the receiving end, another modular multilevel converter, converts power back to ac power. A modular multilevel converter consist of six arms, two for each phase of a three phase system. These converters, however, suffer from the problems of voltage balancing among the arms and circulating current flow in the converter. A study in [1] shows the existence of circulating current in modular multilevel converters. This circulating current affects the performance and efficiency of the converters [2] [3]. Another drawback of modular multilevel converters is the arm voltage balancing as shown in [4]. Variations in voltages of two arms of same phase and between different phases deteriorates the efficiency of the converter [5].

Numerous schemes have been proposed to address these shortcomings. In [6] [7] controls loops are employed to control the internal circulating current and balance the arm's voltages. Another study in [8] uses a single loop to overcome the drawbacks of a two loop control scheme. This scheme, while reducing the complexity of the system, has a trade off with the overall cost of the system. Another approach to deal with circulating current and arm's voltage balancing employs sampling and sorting algorithms [9]. These simple techniques, however, fail to perform in high voltage applications. Another method to control the circulating current employs the control over harmonics in circulating current for its control [10] [11]. Arm's voltage balancing in [12] by employing voltage correcting modules in converter. A divide and conquer approach is followed in [13] to control the internal circulating current in the converter. Moreover, actuators and sensor delays can also be employed to control the circulating current in modular multilevel converters [14]. For improving the steady state and dynamic response of the modular multilevel converter a scheme is adopted in [3]. For balancing the arm's voltages, a scheme is proposed in [5] that preserves the capacitor voltage in sub module when arm's power is varied and simultaneously maintains AC and DC output performance. Arm's voltages can also be balanced by selecting an optimum value for the capacitor of sub module [15]. Redundant voltage levels are employed in [2] to balance the arm's voltages. The arm's voltages and circulating current are also affected when the load is varied or when the load is unbalanced. A study in [15] deals with arm's voltage balancing in unbalanced load conditions.

\section{MULTILEVEL CONVERTER AND ITS MODEL}

Modular multilevel converters were first introduced in [16] and has ever since then been extensively used in energy transmission system and STATCOMS. A three phase modular multilevel converter has six arms, two for each phase. A typical modular multilevel converter is shown in Figure 1. The sub module of the converter acts as a controlled voltage source. Each of the sub module operates in one of three states, i.e. 
State 1, state 2, and dead state as shown in Figure 2 The position of the switches Determine the state of the sub modules as shown in Table 1 Using the switching states, an individual sub module can be inserted or removed from the multilevel converter to increase or decrease the overall voltage of the converter. The voltages of the upper and lower arm are given as

$$
\begin{aligned}
& V_{u g}=n_{u g} \frac{V_{c u g}}{N} \\
& V_{l g}=n_{l g} \frac{V_{c l g}}{N}=\left(N-n_{u g}\right) \frac{V_{c l g}}{N}
\end{aligned}
$$

The upper arm voltage is denoted by Vcug, given by 1 and the lower arm voltage is denoted by Vlg as show in equation 2 . The number of sub modules placed or removed in a phase leg are denoted by nug and nlg for upper and lower arm respectively. Similarly the current of the converter can be divided into three parts
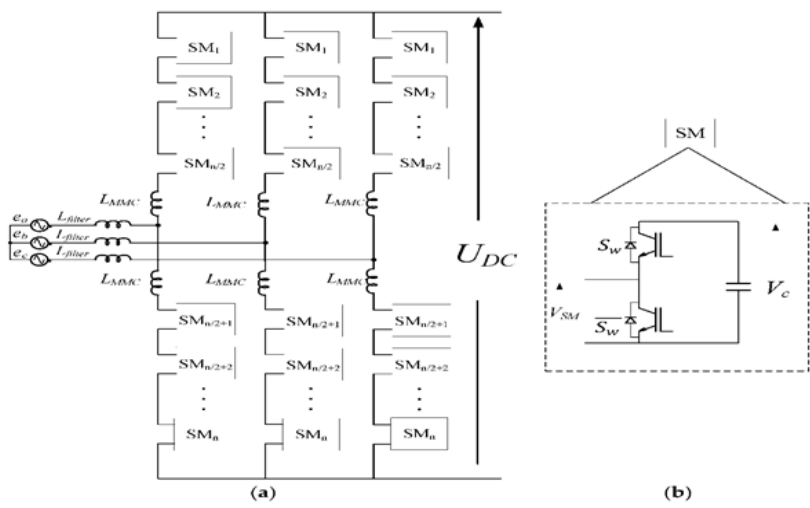

Figure 1. Figure.1 (a)Model of Multilevel Converter (b) sub module of converter

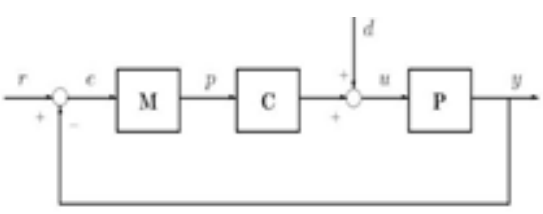

Figure 2. Basic Repetitive controller

$$
\begin{aligned}
& i_{u g}=\frac{i_{g}}{2}+\frac{i_{d c}}{3}+i_{z g} \\
& i_{l g}=-\frac{i_{g}}{2}+\frac{i_{d c}}{3}+i_{z g}
\end{aligned}
$$

Where iug represent the current of the upper arm and ilg represent the current of the lower arm. The circulating current flowing through each is given by Izg. This circulating current is limited to the arms of converter and has no bearings on the converter output voltage but can significantly deteriorates the efficiency of the converter. This circulating current also leads the voltage imbalance between the upper arm and lower arm. Circulating current consist of a dc component and a differential component which are given by

$$
i_{z g}=\frac{i_{u g}+i_{l g}}{2}-\frac{i_{d c}}{3}
$$

TABLE I. SWITCHING STATE OF THE SUB MODULE

\begin{tabular}{l|l|l|l|l|l} 
Mode & $\mathrm{S}_{1}$ & $\mathrm{~S}_{2}$ & $\mathrm{I}$ & $\mathrm{V}_{\text {out }}$ & $\mathrm{dV}_{\mathrm{c}} / \mathrm{dt}$ \\
\hline 1 & Off & On & $>0$ & $\mathrm{~V}_{\mathrm{c}}$ & $>0$ \\
\hline 2 & Off & On & $<0$ & $\mathrm{~V}_{\mathrm{c}}$ & $<0$ \\
\hline 3 & On & Off & $>0$ & 0 & 0 \\
\hline 4 & On & Off & $<0$ & 0 & 0
\end{tabular}

\section{METHODOLOGY}

The proposed scheme for the control of circulating current in modular multilevel converter employs an even harmonic repetitive controller that minimized the even order harmonics and circulating current in the converter. The proposed model consist of a repetitive controller with proportional integral controller. A repetitive controller is a simple controller that is used to track and eliminate a periodic signal in a system. Figure 2 shows a basic repetitive controller. A simple repetitive model consist of a plant, compensator and internal model each represented by transfer function $\mathrm{P}, \mathrm{C}$, and $\mathrm{M}$ respectively. The circulating current in modular multilevel converter also follows a periodic sequence. The Icr in modular multilevel converter consist of two parts as shown in equation 5. The differential current is made of a circulating current component and a dc component. In practical application the dc component is preferred for the stable operation of mmc and for minimizing the losses within mmc. By adjusting the voltage in upper and lower arm the differential component of the circulating current can be controlled. For the control of dc component a novel control scheme is presented here which consist of a combination of even harmonic repetitive controller and a PI controller. The overall control scheme of the proposed model is shown in Figure 3.

The proposed model uses PI controller as a primary mechanism of harmonic suppression of Icr. The Icr reference consist of a dc component, due to total energy, and a fundamental component, due to differential energy. The harmonics of the capacitor easily affects the dc component of the reference current, therefore a moving average filter (MAF) in the path of dc current reference. The arrangement is shown in figure 3 .

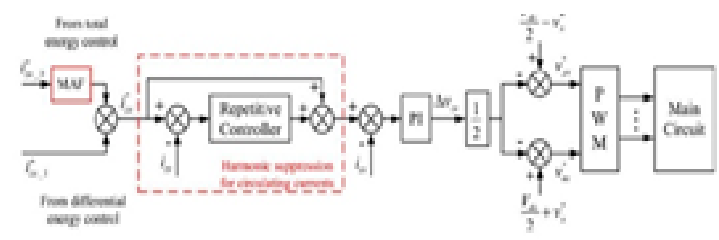

Figure 3. Overall control for internal circulating current

The transfer function of proportional resonant controller is given by

$$
P I(s)=\frac{\left\{K_{p}+K_{i}\right\}}{s}
$$


The Icr equations derived in equation 3 and 4 are used for the derivation of the plant, given by

$$
G(s)=\frac{1}{2 L s}+2 R
$$

The repetitive controller and the PI controller can be combined in different ways to achieve the desired results. In the proposed model we have combined both the systems as shown in Figure 4.

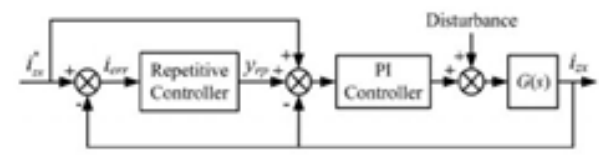

Figure 4. Proposed Repetitive control

\section{Simulation Results}

The proposed model was simulated in Simulink/Matlab. Tabe II shows the specification of the devices used in the converter.

TABLE II. PARAMETERS OF THE CONVERTER FOR PROPOSED MODEL

\begin{tabular}{|c|c|}
\hline Voltage of dc link & $240 \mathrm{~V}$ \\
\hline Output voltage amplitude & $100 \mathrm{~V}$ \\
\hline Load Resistance & $50 \mathrm{ohm}$ \\
\hline Load inductance & $7 \mathrm{mH}$ \\
\hline Output voltage frequency & $50 \mathrm{~Hz}$ \\
\hline Sub modules per arm & 17 \\
\hline Inductance of arm & $4 \mathrm{mH}$ \\
\hline Resistance of arm & $0.5 \mathrm{Ohm}$ \\
\hline Sub module capacitance & $500 \mathrm{micro} \mathrm{farad}$ \\
\hline Gain of Proportional & $\mathrm{Kp}=4, \mathrm{Ki}=8$ \\
\hline controller & \\
\hline Repetitive controller gain & \\
\hline
\end{tabular}

The higher number of levels in the MLCR serve two purposes, i.e. the total harmonic distortion of the system is reduced and the output voltage approaches a sinusoidal waveform. The bode plot of the PI controller is shown in Figure 5.

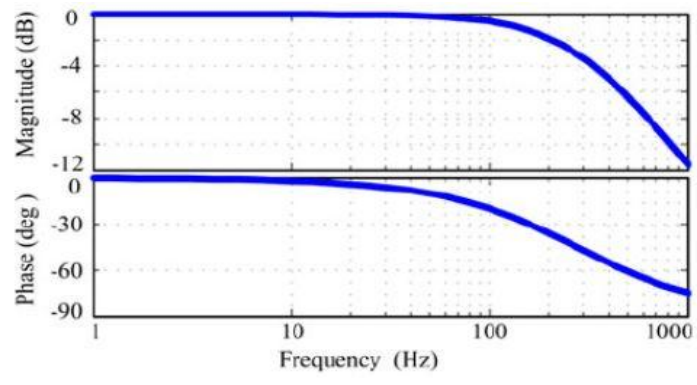

Figure 5. Bode plot of PI Controller

The controller provides unity gain from zero frequency up to the cut off frequency. The frequency is most desirable for the design of the repetitive controller. The cut off frequency of the proportional integral controller needs to be lower than the fundamental frequency in order to remain in the upper portion of the gain curve. From system stability point of view the roots of the controller must be at the origin of unity circle. In the given setup with gain of repetitive controller and PI controller adjusted, the roots lies near to origin as shown in Figure 6.

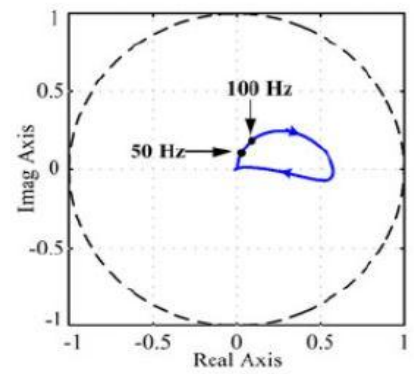

Figure 6. Nyquist Plot of proposed controller

When the system is configured in such a way, it successfully eliminate the even order harmonics from the circulating current. The even order harmonics are shown in Figure 7 without the even harmonics repetitive controller.

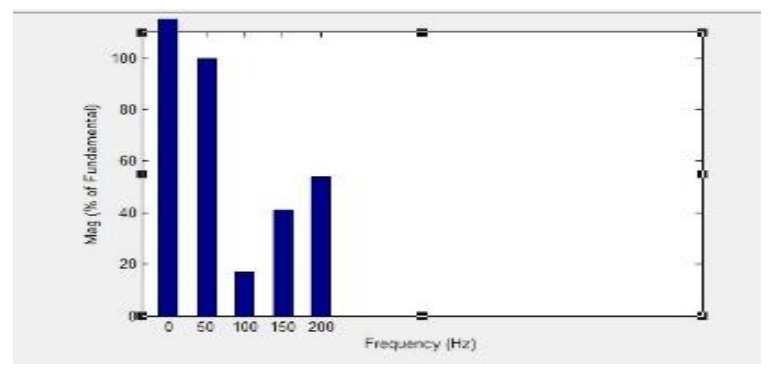

Figure 7. Even order harmonics without proposed controller

The harmonics are very high without the even harmonic repetitive controller. When the controller is inserted in the control loop, the even order harmonics are greatly reduced as shown in Figure 8. 


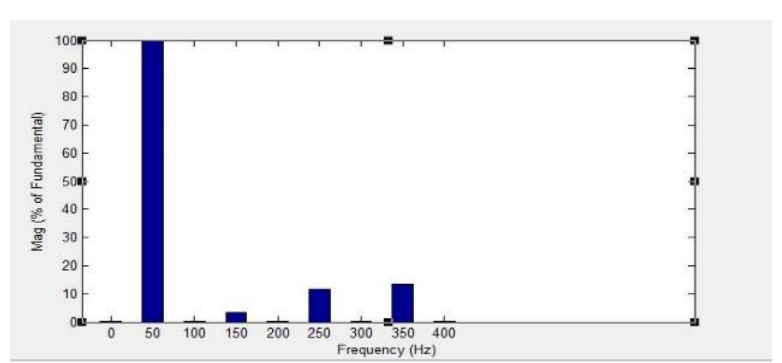

Figure 8. Even order harmonics with the repetitive controller

\section{CONCLUSION}

Modular multilevel converters are the prominent candidates for higher power transmission in future. The robustness and flexibility of the modular multilevel converters also make it the prominent candidate for industrial use. The overarching problem of circulating current can be controlled by using repetitive controllers that are easy to implement and offers increased flexibility in their control. The proposed scheme efficiently reduces the circulating current and even order harmonics as can be seen in the results. The effect of the controller on output voltage is also insignificant as the control is very simple and flexible.

\section{REFERENCES}

[1] L. A. a. H. P. N. A. Antonopoulos, "On dynamics andvoltage control of the modular MLCR," in 13th Eur. Conf. Power Electron. Appl., , Sep. 8-10, 2009,..

[2] J. P. S. C. R. P. J. Z. a. V. G. A. G. Konstantinou, "Control of CCRs in Modular MLCRs Through Redundant Voltage Levels," IEEE Transactions on Power Electronics, vol. 31, no. 11, pp. 7761-7769, Nov. 2016..

[3] Y. W. a. R. Marquardt, "Novel Control Scheme for the Internal Energies and internal circulating current of Modular multilevel converters," in International Exhibition and Conference for Power Electronics, Intelligent Motion, Renewable, PCIM Europe, 2017.

[4] H. L. Z. W. a. T. W. Y. Ma, "Capacitor voltage balancing control of modular MLCRs with energy storage system by using carrier phaseshifted modulation,", in IEEE Applied Power Electronics Conference and Exposition (APEC), , Tampa, FL, 2017.

[5] T. J. S. a. R. E. B. J. D. Stringfellow, "A new arm voltage balancing technique for the control of modular multilevel converter," in 41st Annual Conference of the IEEE Industrial Electronics Society, Yokohama, 2015.

[6] J. P. S. C. J. Z. G. K. a. V. G. A. R. Picas, "Optimal injection of harmonics in CCRs of modular MLCRs for capacitor voltage ripple minimization," in IEEE ECCE Asia Downunder, Melbourne, VIC, 2013.

[7] P. W. Z. C. H. Z. Y. L. a. Y. L. Z. Li, "An Inner Current Suppressing Method for Modular MLCRs," IEEE Transactions on Power Electronics, vol. 28, no. 11, pp. 4873-4879, 2003.

[8] T. G. a. U. K. M. B. S. Riar, "Model Predictive Direct Current Control of Modular MLCRs: Modeling, Analysis, and Experimental
Evaluation," IEEE Transactions on Power Electronics, vol. 30, no. 1, pp. 431-439, 2015.

[9] A. L. a. R. Marquardt, ""An innovative modular MLCR topology suitable for a wide power range," in IEEE Bologna Power Tech Conference Proceedings, 2003.

[10] Y. C. C. T. J. Y. a. X. Y. B. Chen, "Analysis and Suppression of Circulating Harmonic Currents in a Modular MLCR Considering the Impact of Dead Time," IEEE Transactions on Power Electronics, vol. 30, no. 7, pp. 3542-3552, 2015.

[11] A. A. D. S. K. I. M. V. a. H. P. N. L. Angquist, "Open-Loop Control of Modular MLCRs Using Estimation of Stored Energy," IEEE Transactions on Industry Applications, vol. 47, no. 6, pp. 2516-2524, 2011.

[12] B. S. R. a. U. K. Madawala, "Decoupled Control of Modular MLCRs Using Voltage Correcting Modules,", IEEE Transactions on Power Electronics,, vol. 30, no. 2, pp. 690-698, 2015.

[13] M. A. P. D. A. J. R. E. a. J. R. R. Lizana, "Decoupled Current Model and Control of Modular MLCRs," IEEE Transactions on Industrial Electronics, vol. 62, no. 9, pp. 5382-5392, 2015.

[14] S. L. a. M. D. P. Münch, "Modeling and current control of modular MLCRs considering actuator and sensor delays," in 35th Annual Conference of IEEE Industrial Electronics , Porto, 2009.

[15] Z. K. e. al, "Optimal submodule capacitor sizing for modular MLCRs with common mode voltage injection and CCR control," in IEEE Energy Conversion Congress and Exposition (ECCE), Cincinnati,, 2017.

[16] Y. e. a. Zhou, "Analysis and control of modular MLCRs under unbalanced conditions," IEEE Transactions on Power Delivery, pp. 1986-1995, 2013.

[17] A. L. a. R. Marquardt, "An innovative modular MLCR topology suitable for a wide power rang," in IEEE Bologna Power Tech Conference Proceedings, 2003. 